\title{
Interaction between an Anionic Amphiphilic Sequential Polypeptide and Anionic Bilayer Membrane
}

\author{
Masahiro Higuchi, Takatoshi Kinoshita, ${ }^{*}$ Akira Takizawa, \\ Yoshiharu TsuJITA, Kiyomi OKochI, and Naoya HatToRI \\ Department of Materials Science \& Engineering, Nagoya Institute of Technology, \\ Gokiso-cho, Showa-ku, Nagoya 466, Japan
}

(Received June 23, 1990)

\begin{abstract}
The interaction between an anionic amphiphilic sequential polypeptide and an anionic bilayer membrane composed of sodium dilaurylphosphate (DLPNa) was investigated and compared with hydrophobic polypeptide-DLPNa bilayer membrane and anionic polypeptide-DLPNa bilayer membrane interactions. A hydrophobic polypeptide, poly $(\gamma$-methyl L-glutamate) (PMG, $\bar{M}_{v}=4400$ ), was incorporated into the DLPNa bilayer membrane due to their hydrophobic interaction. The incorporation of PMG, however, did not effectively increase sodium ion permeability across the DLPNa bilayer membrane. There was no appreciable interaction between an anionic polypeptide, poly(L-glutamic acid) (PGA, $\bar{M}_{v}=9200$ ), and anionic DLPNa bilayer membrane because of Coulombic repulsion. On the other hand, an anionic amphiphilic sequential polypeptide, poly ( $\gamma$-methyl L-glutamate-co-L-glutamic acid) (am.-MG/GA), of molecular weight 4300 whose $\alpha$-helix was anionic on one face consisting of L-glutamic acid and hydrophobic on the cpposite face comprising of $\gamma$-methyl L-glutamate was easily incorporated into the anionic DLPNa membrane and remarkably increased sodium ion permeability based on a transmembrane bundle consisting of amphiphilic helical rods. It was shown, moreover, that lithium ion apparently decreased the sodium ion permeation through the DLPNa bilayer membrane containing am.-MG/GA.

KEY WORDS Anionic Amphiphilic Sequential Polypeptide / Anionic Bilayer Membrane / Sodium Dilaurylphosphate / Partition / Channel Forming Activity / Intervesicular Adhesion /
\end{abstract}

$\mathrm{We}^{1}$ reported a unique and simple technique for the preparation of an amphiphilic sequential polypeptide (am.-MG/GA), whose sequence is

$$
\begin{aligned}
& \left(G_{A} G_{A} M_{G} M_{G} G_{A} M_{G} M_{G} M_{G} G_{A^{-}}\right. \\
& \left.M_{G} M_{G} G_{A} M_{G} M_{G} M_{G} G_{A} M_{G} M_{G}\right)_{n}
\end{aligned}
$$

where $\mathbf{M}_{\mathbf{G}}$ and $\mathbf{G}_{\mathbf{A}}$ denoted $\gamma$-methyl $\mathrm{L}$ glutamate and L-glutamic acid residue, respectively. A solid condensed monolayer of poly( $\gamma$-methyl L-glutamate) (PMG) at an airwater interface was first formed. The PMG side chains hydrated in the aqueous phase were selectively saponified, keeping the remaining side chains oriented away from the aqueous phase unreacted (Figure 1). As a result, the

\footnotetext{
* To whom correspondence should be addressed.
}

$\alpha$-helix of am.-MG/GA obtained is anionic on one face $\left(G_{A}\right)$ and hydrophobic on the opposite face $\left(\mathrm{M}_{\mathrm{G}}\right)$.

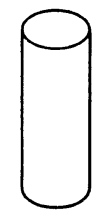

(a)

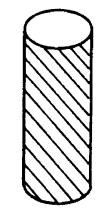

(b)

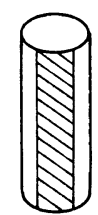

(c)

\footnotetext{
(a) Unshaded cylinder represents a hydrohobic $\alpha$-helix rod (PMG).

(b) Shaded cylinder indicates an anionic $\alpha$-helix $\operatorname{rod}(P G A)$.

(c) An amphiphlic $\alpha$-helix $\operatorname{rod}(\mathrm{am} .-\mathrm{MG} / \mathrm{GA})$ has an anionic face represented by a shaded part.
} 

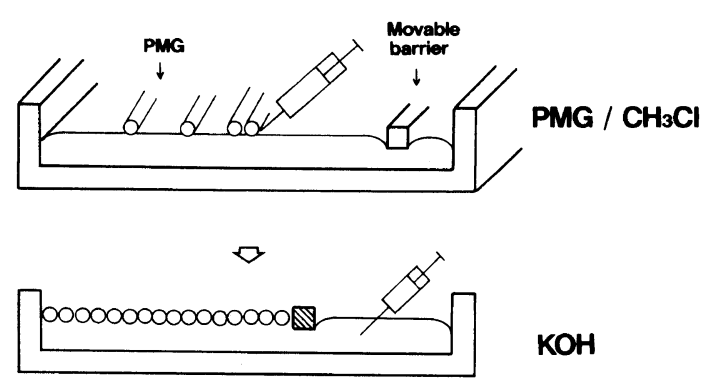

KOH

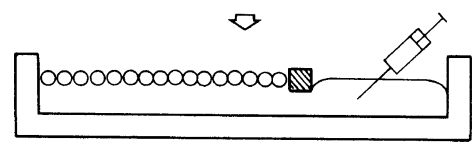

$\mathrm{HCl}$
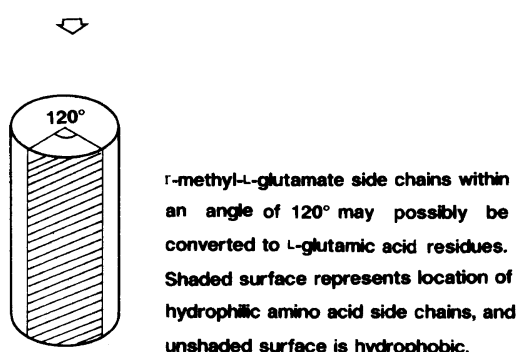

Figure 1. Preparation of an amphiphilic sequential polypeptide, am.-MG/GA.

We report here the interaction between the anionic amphiphilic sequential polypeptide, am.-MG/GA (c), and an anionic bilayer membrane composed of sodium dilaurylphosphate (DLPNa), for comparison with the pure hydrophobic polypeptide, PMG (a)-DLPNa bilayer membrane and pure anionic polypeptide, poly(L-glutamic acid) (PGA) (b)-DLPNa bilayer membrane interactions.

The study of such interactions using artificial membrane systems may give basic insight for understand the structures and functions of native channel membrane proteins. ${ }^{2-8}$

\section{EXPERIMENTAL}

\section{Materials}

Poly( $\gamma$-methyl L-glutamate) (PMG) was obtained by polymerization of $\mathrm{N}$-carboxyanhydride of L-glutamic acid $\gamma$-methyl ester in 1,2-dichloroethane solution with $n$-hex- ylamine as an initiator. ${ }^{9}$ The molar ratio of the anhydride to initiator was 75. Polymerization occurred at room temperature for $24 \mathrm{~h}$. The PMG obtained was precipitated in dry methanol. A molecular weight of 4400 was estimated from viscosity measurements in dichloroacetic acid.

Poly(L-glutamic acid) (PGA, $\left.\bar{M}_{v}=9200\right)$ was kindly provided by Ajinomoto Co., Ltd.

The preparation of an amphiphilic sequential copolypeptide consisting of $\gamma$-methyl Lglutamate and L-glutamic acid (am.-MG/GA) was previously reported in detail in another paper. ${ }^{1}$ The preparation method of am.-MG/ GA is outlined in Figure 1. A known amount of PMG dissolved in chloroform was placed at the air-water interface in a L-B trough of moving wall type ${ }^{10}$ (Nippon Laser \& Electronics Lab., NL-LB240-MWA) with a syringe. The solution spread over the interface and the solvent evaporated leaving the PMG monolayer. The area occupied by the monolayer decreased by moving a hydrohobic barrier to form the solid condensed state of the monolayer. When the area per monomer residue of PMG reached to $15 \AA^{2}$, a $2.5 \mathrm{M}$ aqueous solution of potassium hydroxide $(\mathrm{KOH})$ was inserted in large excess into the aqueous phase beneath the solid condensed monolayer. The molar ratio of $\mathrm{KOH}$ to $\gamma$-methyl L-glutamate residue was $10^{5}$. After ca. $10 \mathrm{~min}$, excess hydrochloric acid was added to convert the copolypeptide to its acidic form. Hydrolysis of the resulting copolypeptide, am.-MG/GA, by Staphylococcus aureus, Strain V8 protease, and gel filtration chromatographic analysis ${ }^{1}$ showed that the sequence of am.-MG/GA is

$$
\begin{aligned}
& \left(\mathrm{G}_{\mathrm{A}} \mathrm{G}_{\mathrm{A}} \mathrm{M}_{\mathrm{G}} \mathrm{M}_{\mathrm{G}} \mathrm{G}_{\mathrm{A}} \mathrm{M}_{\mathrm{G}} \mathrm{M}_{\mathrm{G}} \mathrm{M}_{\mathrm{G}} \mathrm{G}_{\mathrm{A}^{-}}\right. \\
& \left.\mathrm{M}_{\mathrm{G}} \mathrm{M}_{\mathrm{G}} \mathrm{G}_{\mathrm{A}} \mathrm{M}_{\mathrm{G}} \mathrm{M}_{\mathrm{G}} \mathrm{M}_{\mathrm{G}} \mathrm{G}_{\mathrm{A}} \mathrm{M}_{\mathrm{G}} \mathrm{M}_{\mathrm{G}}\right)_{n} .
\end{aligned}
$$

Therefore, when am.-MG/GA forms $\alpha$-helical structure, an amphiphilic rod can be made, i.e., anionic on one face consisting of L-glutamic acid $\left(G_{A}\right)$ side chains and hydrophobic on the opposite face comprising of $\gamma$-methyl $\mathrm{L}$ glutamate $\left(\mathrm{M}_{\mathrm{G}}\right)$ side chains. 


\section{Fluorescent Probes}

PMG containing $2.7 \mathrm{~mol} \%$ of 1 -amino-8anilinonaphthalene (AN) in the side chain (AN-PMG) was obtained by a condensation reaction of $\operatorname{poly}(\gamma$-methyl L-glutamate- $c o$-Lglutamic acid) containing $3 \mathrm{~mol} \%$ of $\mathrm{L}-$ glutamic acid, ${ }^{11}$ with $\mathrm{AN}$ in dimethylformamide using dicyclohexylcarbodiimide (DCC) and $n$-hydroxybenzotriazole $(\mathrm{HOBt})$ for $24 \mathrm{~h}$ at room temperature. ${ }^{12}$ PGA and am.-MG/GA with $3 \mathrm{~mol} \%$ of $\mathrm{AN}$ in the side chains were obtained in the same way as AN-PMG. It should be noted that, in the fluorescent modification of am.-MG/GA, an AN group was necessarily introduced to the anionic face of the amphiphilic $\alpha$-helix rod. The content of $\mathrm{AN}$ in the polypeptides was determined by fluorescence spectroscopic analysis.

1-Anilino-8-propionamide-naphtalene (ProAN) as a model compound of the AN modified polypeptides was synthesized by the condensation reaction between propionic acid and $\mathrm{AN}$ using DCC and HOBt.

DLPNa vesicle solution containing am.MG/GA labeling $\mathrm{NH}_{2}$-terminal with 7-chloro4-nitrobenzo-2-oxa-1,3-diazol $(\mathrm{NBD})^{13}$ for fluorescence microscopic measurements was prepared as follows. A DLPNa vesicle solution, $10 \mathrm{ml}$, containing am.-MG/GA $(6.0 \mu \mathrm{g})$ was incubated at $60^{\circ} \mathrm{C}$ with $10 \mu \mathrm{l}$ of ethanol solution of NBD $\left(10^{-2} \mathrm{M}\right)$ for $1 \mathrm{~min}$. After the reaction, the solution was rapidly quenched to $0^{\circ} \mathrm{C}$. The resulting DLPNa vesicle containing am.$\mathrm{MG} / \mathrm{GA}$ carrying $\mathrm{NBD}$ at the $\mathrm{NH}_{2}$-terminal was observed to have strong fluorescence.

\section{Amphiphile}

Dilaurylphosphate was kindly provided by Kao Co., Ltd. Sodium dilaurylphosphate (DLPNa) was obtained by neutralization of dilaurylphosphate with $\mathrm{NaOH}$ and the following recrystallization from acetone.

\section{Vesicles}

The DLPNa vesicles containing polypeptides (PMG and am.-MG/GA) were prepared as follows. PMG and DLPNa were each dissolved in chloroform and am.-MG/GA was dissolved in dimethylformamide. These solutions of polypeptides and DLPNa were mixed and poured into a glass flask, and then a thin film was formed in the inner surface of the flask by evaporating the solvent. $50 \mathrm{~mm}$ Tris-HEPES buffer solution, $\mathrm{pH}$ 6.8, was added to this flask, and it was sonicated at $0^{\circ} \mathrm{C}$ under a stream of nitrogen by using a Bionic model 7250B ultrasonic processor (SEIKO I \& E/ Sonic \& Materials), for $10 \mathrm{~min}$ to prepare the vesicles. PGA was directly added to the buffer solution of DLPNa before sonication. The molar ratio of polypeptides to DLPNa was fixed at $3.1 \times 10^{-4}$. The phase transition temperature of DLPNa vesicle was $25^{\circ} \mathrm{C}$ from differential scanning calorimetry.

\section{METHODS}

\section{Spectroscopic Measurements}

Fluorescence spectra of Pro-AN and AN modified polypeptides were obtained with a spectrofluorophotometer (Shimadzu, RF-540). The excitation wavelength of AN was $350 \mathrm{~nm}$ and the concentration of DLPNa was 0.2 $\mathrm{mg} \mathrm{ml}^{-1}$.

\section{Microscopic Measurements}

The shape of the DLPNa vesicles was directly observed with a phase-contrast microscope (Olympus Optical, Model BHR, $\times 1000$ ). The concentration of DLPNa was $0.2 \mathrm{mg} \mathrm{ml}^{-1}$.

Fluorescence microscopic measurements of DLPNa vesicles containing am.-MG/GA with $\mathrm{NBD}$ at the $\mathrm{NH}_{2}$-terminal, were carried out using a fluorescence microscope (Olympus Optical, Model BHS-RFK, $\times 1250)$. The concentration of DLPNa was $0.2 \mathrm{mg} \mathrm{ml}^{-1}$. NBD was excited by a super high pressure mercury lamp using BP490 and EY455 filters (Olympus Optical), and fluorescence emission was detected by a Olympus camera PM10ADS. 


\section{Permeation Measurements}

DLPNa vesicle solution containing potassium gluconate in the interior was prepared as follows. DLPNa $(50 \mathrm{mg})$ was added to the $50 \mathrm{mM}$ Tris-HEPES buffer, $\mathrm{pH} 6.8,(5 \mathrm{ml})$ containing potassium gluconate $(0.1 \mathrm{M})$, and it was sonicated in a similar manner as above. $1 \mathrm{ml}$ of the vesicle solution thus obtained was then passed through a Bio-gel P-6DG gel filtration column $(10 \phi \times 400 \mathrm{~mm})$ (Bio-Rad Lab.) by using $0.1 \mathrm{M}$ sodium gluconate in $50 \mathrm{mM}$ Tris-HEPES buffer, $\mathrm{pH} 6.8$, as a eluting buffer to remove the external potassium gluconate. The fraction containing DLPNa vesicles was collected and added to the buffer solution containing $0.1 \mathrm{M}$ sodium gluconate. The total volume was $50 \mathrm{ml}$. The resulting vesicles had an ionic concentration gradient between the interior ( $0.1 \mathrm{M}$ potassium ion) and exterior $(0.1 \mathrm{M}$ sodium ion) under isotonic condition. The addition of polypeptides to the vesicle was performed by the addition of dimethylformamide solution of polypeptides $\left(1 \mathrm{mg} \mathrm{ml}^{-1}\right)$. The amount of polypeptide added was $7.1 \times 10^{-9} \mathrm{~mol}$. The amounts of potassium ions transported across the vesicular bilayer before and after the addition of polypeptide, were determined with an ion meter (Horiba Co., Ltd., N-7ionII) at $20^{\circ} \mathrm{C}$. In this case, the vesicular external sodium ion having larger hydration size than that of the internal potassium ion determined the permeability across the membrane. Furthermore, because of electroneutrality the permeability of potassium ion from the vesicular interior to the external solution was the same as that of sodium ion from the external to the interior.

\section{RESULTS AND DISCUSSION}

\section{Partition of Polypeptides in DLPNa Bilayer Membrane}

We already reported ${ }^{14}$ interactions between polypeptides and a cationic bilayer membrane consisting of distearyldimethylammonium chloride (DSACl), indicating that the hydro-

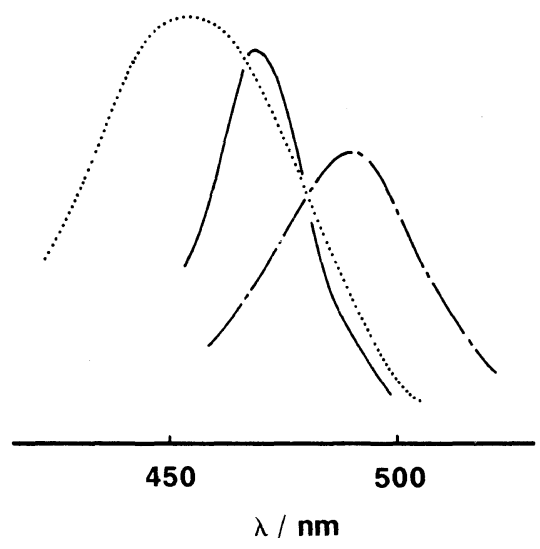

Figure 2. Fluorescence spectra of AN modified polypeptides in DLPNa bilayer membrane: ( $\cdots)$, AN-PMG; $(---)$, AN-PGA; (-), AN-am.-MG/GA; [DLPNa] $=0.02 \mathrm{wt} \%$; [polypeptide] $/[\mathrm{DLPNa}]=3.1 \times 10^{-4}$.

phobic polypeptide, PMG, was readily incorporated into the hydrophobic membrane interior to form membrane-spanning helix. However, the negative charged polypeptide, PGA, can hardly penetrate through the membrane owing to the electrostatic interaction between the anionic PGA and the cationic membrane surface, resulting in the formation of cross-links between vesicles. We elucidated, here, the partition of polypeptides in the anionic bilayer membrane consisting of DLPNa by means of fluorescence spectroscopic measurements. It is well known that fluorescence characteristics, such as emission maxima and fluorescence intensity of anilinonaphthalene derivatives are very sensitive to environmental polarity around the fluorescence probes. ${ }^{15}$ Figure 2 shows the fluorescence spectra of $A N$ modified polypeptides (AN-PMG, AN-PGA and $\mathrm{AN}$-am.-MG/GA) in DLPNa vesicle solution. The relationship between the empirical solvent polarity, $Z,{ }^{16}$ and the emission maxima, $\lambda_{\max }$, of AN modified polypeptide in DLPNa vesicle solution is shown in Figure 3. The solid line in the figure shows the relationship between the $Z$ value and $\lambda_{\max }$ of Pro-AN, the fluorescent model compound for AN modified polypeptides, in various solvents. 


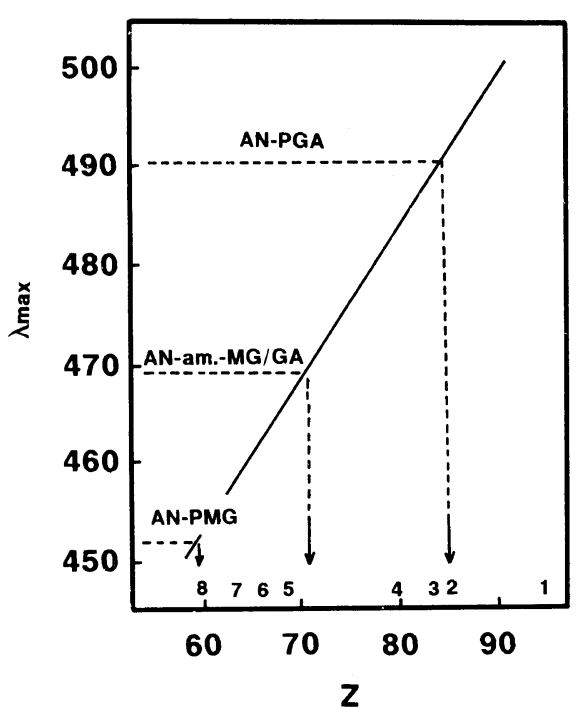

Figure 3. Relation between fluorescent emission maxima of Pro-AN and empirical $Z$ solvent polarity. [Pro-AN] = 3.0 × $10^{-5} \mathrm{M}$; (1), water; (2), methanol; (3), ethanol; (4), 2-propanol; (5), dimethylformamide; (6), acetone; (7), chloroform; (8), octane.

It is apparent that the $\lambda_{\max }$ of Pro-AN shifts to higher wavelength with increasing solvent polarity. ${ }^{17}$ The $\lambda_{\text {max }}$ of AN-PMG, hydrophobic polypeptide, in DLPNa vesicle solution, $453 \mathrm{~nm}$, corresponds to the $Z$ value of octane. However, the $\lambda_{\text {max }}$ of AN-PGA, anionic polypeptide, in DLPNa vesicle solution, $490 \mathrm{~nm}$, corresponds to the $Z$ value of methanol. Moreover, the $\lambda_{\max }$ of AN-PGA in the vesicle solution coincided with that of AN-PGA in the buffer solution without vesicles. Therefore, the hydrophobic PMG may readily be incorporated into the DLPNa membrane interior in a similar manner as the cationic membrane system, ${ }^{14}$ whereas the negatively charged PGA can hardly interact with negatively charged DLPNa and this is present in the aqueous phase outside the vesicle. On the other hand, the $\lambda_{\max }$ of AN-am.MG/GA in DLPNa vesicle solution, $468 \mathrm{~nm}$, corresponds to $Z$ values between AN-PMG (hydrophobic membrane interior) and ANPGA (aqueous phase outside the vesicle). As described in the experimental section, the

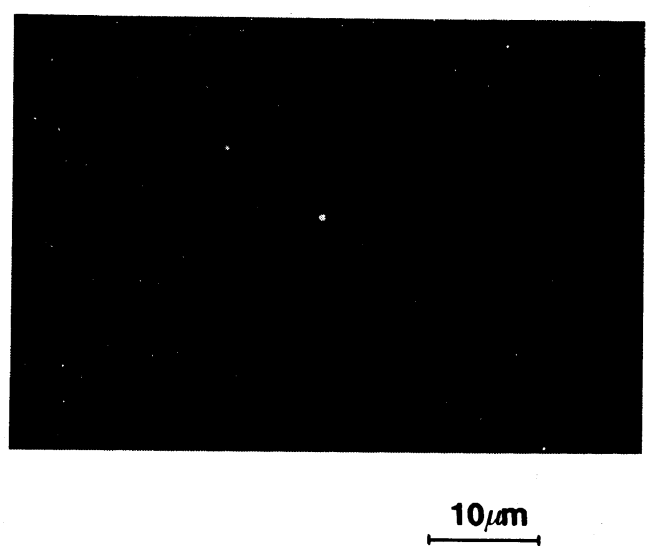

Figure 4. Fluorescence micrograph of DLPNa vesicles containing am.-MG/GA labeled at the $\mathrm{NH}_{2}$-terminal with $\mathrm{NBD}$, initial magification $\times 1250$ : [DLPNa] $=0.02 \mathrm{wt} \%$; [polypeptide] $/[\mathrm{DLPNa}]=3.1 \times 10^{-4}$.

fluorescent AN group was localized at the anionic face of the amphiphilic $\alpha$-helix rod. Therefore, the $\lambda_{\max }$ of $468 \mathrm{~nm}$ means that the anionic face of $\mathrm{AN}$-am.-MG/GA may possibly be accessible to neither the aqueous phase nor the hydrocarbon chains of DLPNa. A simple understanding of the intermediate polarity around the anionic face of am.-MG/GA is that in the DLPNa membrane phase several am.-MG/GA molecules associate to form a transmembranous bundles in which the anionic faces are in contact with each other.

Further, in the fluorescence micrograph of DLPNa vesicles containing am.-MG/GA labeled with NBD, the fluorescence emission of NBD could be observed as spherical particles (vesicles) on the dark background (aqueous phase) (Figure 4). This may support the incorporation of am.-MG/GA into the anionic bilayer membrane. These results suggest the following possibilities concerning the partition of am.-MG/GA in DLPNa bilayer membrane.

(1) The anionic am.-MG/GA can be incorporated into the anionic DLPNa membrane due to the formation of transmembranous bundles composed of several am.MG/GA molecules.

(2) The hydrophilic (anionic) faces, which 
are accessible to neither the aqueous phase nor the hydrocarbon region of the membrane, are in contact with each other in the interior of the transmembrane bundle.

(3) The hydrophobic residues of each am.-MG/GA are on the exterior surface of the transmembrane bundle in contact with the hydrocarbon region of DLPNa membrane.

\section{A Channel Forming Activity of Polypeptides} Incorporated into the DLPNa Bilayer Membrane Vesicle

Figure 5 shows the rate of sodium ion permeation through the bilayer membrane of DLPNa vesicle from the vesicular exterior to the interior. The permeabilities of sodium ion through the bilayer membrane were measured before and after the addition of dimethylformamide solution of polypeptides, respectively, at $20^{\circ} \mathrm{C}$. The pure dimethylformamide did not induce any change in the rate of the sodium ion permeability. Sodium ion permeability was independent of the addition of PGA, since PGA, as noted above, could not interact with DLPNa vesicle owing to the electrostatic repulsion. On the other hand, the addition of PMG and am.-MG/GA, both having interac- tion with DLPNa, induced an increase in sodium ion permeability. However, the degree of increase in permeability induced by the addition of am.-MG/GA was shown to be much larger than that by the addition of PMG.

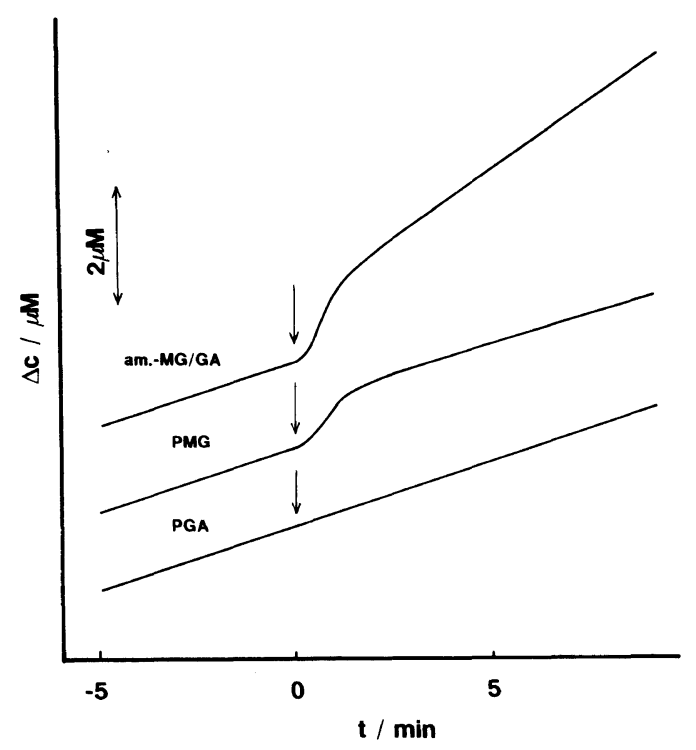

Figure 5. Effects of polypeptides, PMG, PGA and am.-MG/GA, addition on the rate of sodium ion permeation through the bilayer membrane consisting of DLPNa at $20^{\circ} \mathrm{C}$. The arrow marks the time at which the polypeptides were introduced.

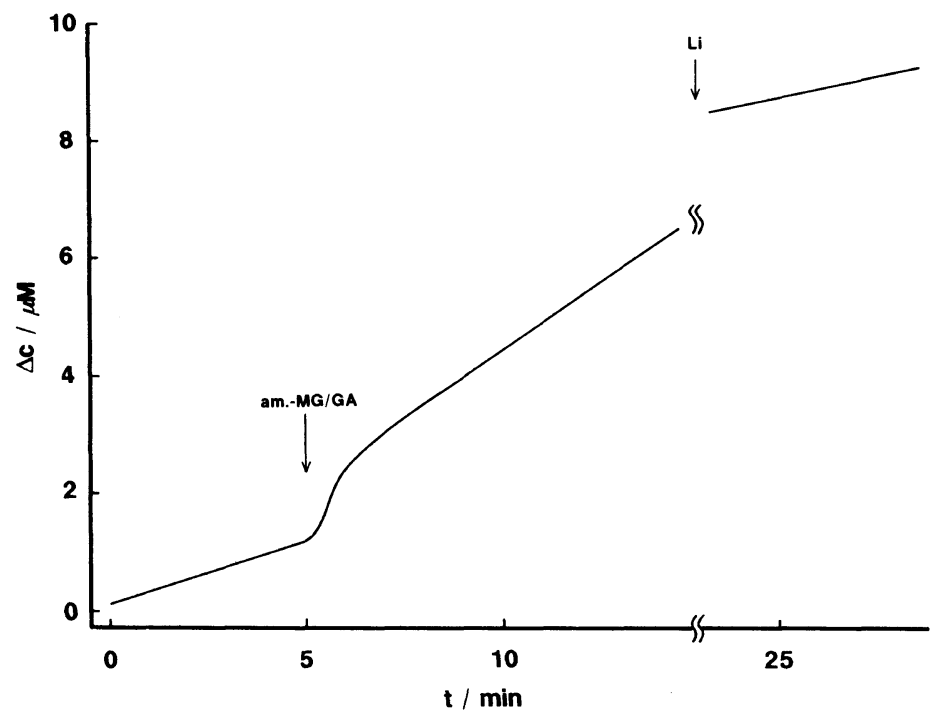

Figure 6. Effect of lithium ion on the rate of sodium ion permeation through DLPNa bilayer membrane containing am.-MG/GA at $20^{\circ} \mathrm{C}$. The arrow indicates the time at which the lithium ion was introduced. 
This may be explained in terms of the hypothetical molecular arrangement of am.$\mathrm{MG} / \mathrm{GA}$ in the bilayer membrane based on the fluorescent and microscopic studies. That is, am.-MG/GA aggregate with each other to form transmembrane bundles having an hydrophobic exterior and hydrophilic inner pores surrounded by anionic surface. Through the anionic pores cations may be transported.

On the other hand, the slight increase in permeability through the anionic membrane with PMG, inspite of the easy incorporation of PMG into the membrane, is associated with the absence of the ionic permeation site in PMG. ${ }^{14}$

Furthermore, the ionic permeability through DLPNa membrane was strongly dependent on the addition of lithium ion. Figure 6 shows the lithium ion induced changes in sodium ion permeability through DLPNa bilayer membrane containing am.-MG/GA. The permeability of sodium ion through the bilayer membrane decreased by a factor of 3 , on adding $1.5 \times 10^{-5} \mathrm{M}$ lithium ion. The shape of the vesicle obtained by sonication was essentially spherical (Figure 7a). However, the addition of lithium ion produced intervesicular adhesion (Figure $7 b$ ). It has been shown ${ }^{18}$ that the coordination number of lithium ion is 4 and the stability constant of lithium ion-phosphoric acids (head groups of the vesicle) complex, 436, is much greater than that of sodium ion (61) and potassium ion (51). Therefore, one interpretation is that intervesicular adhesion may be induced by a cross-linking bridge between DLPNa vesicles owing to complexation of lithium-phosphoric acid head groups of DLPNa vesicle, although evidence for this complex formation was not experimentally obtained. In any event, it is evident that the channel forming activity of am.-MG/GA in the anionic DLPNa membrane is apparently changed by the addition of lithium ion via vesicular structure change (Figure 7(b)).

Acknowledgment. We acknowledge the fi-

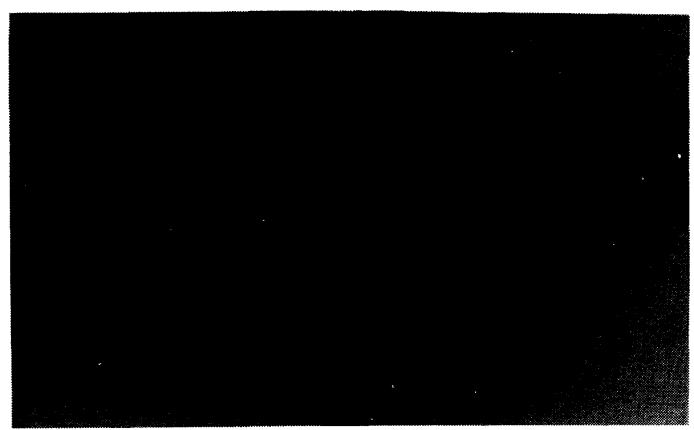

(a)

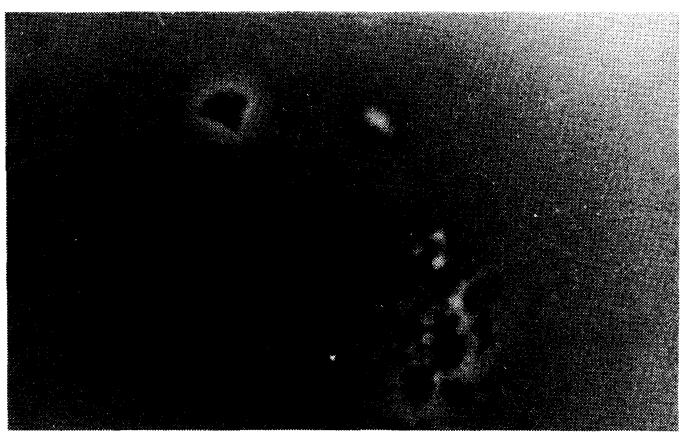

(b)

$10 \mu \mathrm{m}$

Figure 7. Phase-contrast micrographs of DLPNa vesicles (a); in $0.1 \mathrm{M}$ sodium gluconate solution and (b); $0.1 \mathrm{M}$ lithium gluconate solution, initial magnification $\times 1000$; $[\mathrm{DLPNa}]=0.02 \mathrm{wt} \%$.

nancial support of a Grant-in-Aid from the Ministry of Education, Science, and Culture of Japan.

\section{REFERENCES}

1. M. Higuchi, T. Kinoshita, A. Takizawa, Y. Tsujita, and K. Okochi, Macromolecules, 23, 361 (1990).

2. O. F. Jr. Robert and M. R. Frederic, Nature, 300, 325 (1982).

3. D. W. Urry, T. L. Trapane, and K. U. Prasad , Science, 221, 1064 (1983).

4. M. T. Tosteson and D. C. Tosteson, Biophys. J., 36, 109 (1981).

5. H. Vogel and F. Jahnig, Biophys. J., 50, 573 (1986).

6. M. Noda, H. Takahashi, T. Tanabe, M. Toyosato, S. Kikyotani, Y. Furutani, T. Hirose, H. Takashima, 
S. Inayama, T. Miyata, and S. Numa, Nature, 302, 528 (1983).

7. J. F. Moor and R. M. Stroud, Proc. Natl. Acad. Sci. U.S.A., 81, 155 (1984).

8. P. R. Schofield, M. G. Darlison, N. Fujita, D. R. Burt, F. A. Stephenson, H. Rodriguez, L. M. Rhee, J. Ramachandran, V. Reale, T. A. Glencorse, P. H. Seeburg, and E. A. Barnard, Nature, 326, 221 (1987).

9. C. H. Banford, A. Elliot, and W. E. Hanby, "Synthetic Polypeptides," Academic .Press, New York, N.Y., 1956.

10. H. Kumehara, T. Kasuga, T. Watanabe, and S. Miyata, 4th International Conference on L-B Films, 1989, p. 226.

11. A. Takizawa, M. Higuchi, T. Kinoshita, and Y.
Tsujita, Colloid. Polym., 265, 31 (1987).

12. J. L. Houben, A. Fissi, D. Bacciola, N. Rosato, O. Pieroni, and F. Ciardelli, J. Biol. Macromol., 5, 94 (1983).

13. P. B. Ghosh and M. W. Whitehouse, Biochem. J., 108, 155 (1968).

14. M. Higuchi, T. Kinoshita, A. Takizawa, and Y. Tsujita, Polym. J., 21, 295 (1989).

15. G. K. Radda, Biochem. J., 122, 385 (1971).

16. E. M. Kosower, J. Am. Chem. Soc., 80, 3253 (1958).

17. D. C. Turnner and L. Brand, Biochemistry, 7, 3381 (1967).

18. R. W. Hay, "Bio-inorganic Chemistry," Ellis Horwood, England, 1984. 